\title{
CAPERUCITA ROJA O LOS COMPORTAMIENTOS INADECUADOS. LOS ARQUETIPOS Y LA LITERATURA ORAL EN EL AUDIOVISUAL Y LA GENERACIÓN DE PENSAMIENTO ${ }^{1}$
}

\author{
Yolanda Cruz López ${ }^{2}$
}

Caperucita Roja o los comportamientos inadecuados. Los arquetipos y la Literatura Oral en el audiovisual y la generación de pensamiento

Resumen: El argumentario cinematográfico se nutre de la literatura, dado que esta se origina en la tradición oral, los arquetipos literarios primitivos están presentes en el cine y, a través de este, educan y general pensamiento. Se han desarrollado dos unidades didácticas para alumnado de Secundaria y para una asociación de mujeres, en torno al arquetipo de Caperucita Roja con el cortometraje "Una nit", de Marta Bayarri. Los resultados alcanzados confirman que el desconocimiento del lenguaje audiovisual reduce a espectadoras y espectadores a la pasividad, aceptando actitudes normalizadas y negativas en la búsqueda de su autoaceptación y autoafirmación en igualdad.

Palabras clave: Arquetipo, Caperucita Roja, cine, cortometraje, igualdad, competencia audiovisual.

Little Red Riding Hood or Improper Behaviours. Archetypes and Oral Literature in the Audio-Visual Media and their Role in Generating Thought

Abstract_: The cinematographic argument is nourished by literature, since the literature originates in the oral tradition, the primitive literary archetypes are present in the cinema and from it they educate and generate thought. Two didactic units have been developed for Secondary Education students and for an association of women, around the archetype of Little Red Riding Hood with the short film "Una nit", by Marta Bayarri. The results achieved confirm that ignorance of audio-visual language reduces spectators to passivity by accepting normalized and negative attitudes in the search for self-acceptance and self-affirmation in equality.

Key words: Archetype, Little red Hood, film, short film, equality, audio-visual competence.

\section{Introducción}

Los medios audiovisuales facilitan un recurso didáctico en la enseñanza de la literatura y reportan valiosos resultados por captar de manera rápida y eficaz la atención del alumnado. Sin embargo, ni en el mundo académico ni en la vida cotidiana, la manifiesta y profunda atracción que siente el público por los medios audiovisuales, así

\footnotetext{
${ }^{1}$ Fecha de recepción: 15/05/2019.

Fecha de aceptación: 08/03/2020.

2 Profesora del Máster en Comunicación Social, Universidad de Almería; miembro del Grupo de Investigación "Mujeres, Literatura y Sociedad (HUM874) y del CYSOC, UAL; $\square$ yolandacru@gmail.com
} 
como el interés que pueda tener el profesorado a la hora de usarlos, son directamente proporcionales ni a la existencia de un material didáctico audiovisual adecuado, ni a la calidad de éste. Observada la base de datos del Ministerio de Cultura ${ }^{3}$, desde marzo de 2011 a agosto de 2016, en nuestro país se estrenaron 337 películas españolas, de las que cerca del $12 \%$ son adaptaciones o versiones libres de obras literarias o de argumentos inspirados en fuentes de tradición oral. Estas películas y las producciones internacionales estrenadas en las salas de cine españolas podrían servir de herramienta didáctica e informativa para acercar al alumnado, en un ámbito académico, y a la sociedad, en general, a la tradición literaria oral.

Sin embargo, el uso del cine (películas, documentales, cortometrajes, animación, video-creación, etc.) como instrumento didáctico de la literatura ha de realizarse correctamente, y para ello se precisa la elaboración de una secuencia didáctica previa y posterior al visionado, así como una estudiada selección de textos cinematográficos a los que acudir en cada caso.

La Literatura Oral podría ser definida como el apéndice de homologías estructurales de Literatura que se transmite esencialmente a través de la palabra, aunque puede tener también algún tipo de transmisión escrita subsidiaria, como la literatura de cordel. Existen estudios relacionados con la literatura y el cine, pero referidos a la literatura de autor o a la literatura culta. Los estudiantes reciben una educación literaria basada, principalmente, en la historia de la literatura: autores, fechas y obras que deben ser memorizadas para considerarles personas formadas literariamente. Sin embargo, se obvia que el origen de la literatura no coincide con la aparición del primer libro escrito, sino que se trata, probablemente, de un acontecimiento prehistórico y preliterario. Por tanto, de la estética de la oralidad bien pudiera haber surgido el primer texto literario y

\footnotetext{
${ }^{3}$ La fuente utilizada para aportar ese dato es la página web del Ministerio de Cultura (www.mcu.es)
} 
siendo un poco más osados se podría añadir que, si así fue, es muy probable que también surgiera el primer relato fílmico. El extenso corpus de trabajos de investigación literaria y cinematográfica existente sobre las interrelaciones entre ambas a nivel argumental y de lenguaje, se completaba hace unos años, con la publicación de Biblioteca del cine español (Heredero y Antonio 2010), ya un instrumento imprescindible a la hora de estudiar las relaciones de la literatura española y universal con el cine español. Heredero y Santamarina, catalogan las obras cinematográficas de producción nacional y coproducciones con otros países comprendidas entre 1900, fecha de la primera cinta identificada con raíces literarias y 2005, fecha de finalización de la investigación que realizaron ambos críticos. Hasta la publicación de esta Biblioteca y tal y como reconocen sus autores (2010:12), los catálogos elaborados por Gómez (1978) y Quesada (1986), así como las relaciones precedentes; Alba (1999) para el Ministerio de Asuntos Exteriores, Escudero Cuesta (2005) para la Comunidad Autónoma de Madrid (CAM) y la base de datos de Camarero (2006) para la Biblioteca Virtual Miguel de Cervantes, solo recogían obras literarias y autores considerados de calidad y prestigio. Heredero y Santamarina (2010) dan un paso adelante ya que a la hora de buscar las fuentes de los argumentos de las producciones audiovisuales tiene en cuenta "todas las formas literarias posibles" (2010:13), para ellos; la novela, el teatro, los relatos breves, las canciones y romances, en español o en cualquier otra lengua.

Sin entrar a valorar las dificultades que presenta este tipo de investigación reconocidas por los autores, tales como la dificultad a la hora de constatar la autoría de algunos guiones u obras, ni los criterios por los que la Biblioteca deja fuera de catálogo las producciones audiovisuales de menos de 60 minutos, sí que para la investigación de la que forma parte el presente artículo, partimos de la nula atención que ambos prestan en la obra a los cuentos de tradición oral y a las leyendas como fuentes argumentales, 
así como a la necesidad de reclamar un lugar para ellas, por tratarse de etnotextos de comprobada influencia en la literatura escrita posterior.

El presente artículo es resultado de una investigación más amplia cuya primera etapa partió de la selección de una serie de cuentos, leyendas y leyendas urbanas recopilados en distintos trabajos científicos de contenido etnográfico (Pedrosa 2004; Gómez, Martínez y Pedrosa 2007; Gómez y Pedrosa 2002; Morote 2010; Gómez 1998), para, a partir de ahí, buscar versiones y adaptaciones cinematográficas de sus argumentos y realizar un estudio intertextual entre la literatura oral y el cine. Posteriormente, nos ceñimos al estudio de etnotextos o transcripciones fieles de literatura oral, catalogados como cuentos maravillosos o de hadas, para estudiar la presencia de estos y de los arquetipos femeninos que los sustentaban en el cine. La investigación se completó con la elaboración de una base de datos de cortometrajes con los que trabajar de modo académico, divulgativo y terapéutico, utilizando, entre otros, el material recibido por el festival Visualízame, Audiovisual \& Mujer, que organiza Fundación Inquietarte en sus seis primeras ediciones, como parte de un proyecto UNESCO, coordinado por la directora de la Cátedra de Economía Solidaria de la Universidad Abat Oliba CEU, Carmen Parra.

La elección del formato cortometraje se deriva de las posibilidades que ofrece a la hora de trabajarlo en sesiones didácticas tanto por la corta duración del texto fílmico, como por el ritmo narratológico empleado por las realizadoras y realizadores, más cercano al ritmo de los productos audiovisuales que consume la franja de población cuya edad oscila entre los 18 y los 28 años, integrada por personas jóvenes inmersas en la omnipresencia mediática de la Sociedad de la Información, (Trejo 2001) y que se caracterizan por ser desconocedoras del lenguaje audiovisual, fácilmente influenciadas a la hora de empatizar con los roles con los que se identifican y a través de los que 
desarrollan sus relaciones con la sociedad, y necesitadas de adquirir una competencia audiovisual que les permita mantener su mirada y fomentar su espíritu crítico (Parra y Cruz 2015: 267-278).

La presente investigación muestra el desarrollo de una unidad didáctica diseñada en relación con el cortometraje "Una nit" (Bayarri 2013) con alumnado de $4^{\text {o }}$ de la ESO (Instituto Sierra Magina de Huelma, Jaén) y con mujeres de la asociación Nuevas Formas (Íllar, Almería) quienes, posteriormente, mediante encuesta pudieron valorar tanto los contenidos argumentales analizados durante la lectura del texto fílmico como el uso de este como método y recursos educativo.

1.1. Objetivos generales de la presente investigación

El objetivo principal es el de conocer las posibilidades del audiovisual aplicado a la formación siendo este el principal transmisor de información, conocimiento e ideología en la Sociedad de la Información en cuyo seno nos relacionamos y desarrollamos. La capacidad del cine para generar emociones y el uso de estas para facilitar la adquisición de valores y conocimiento.

\subsection{Objetivos específicos}

El objetivo descrito anteriormente implica una hoja de ruta diseñada desde una perspectiva de género con los siguientes objetivos específicos:

- Análisis de las consecuencias de la revolución tecnológica en el campo de la comunicación y en el de la formación, así de la imagen de la mujer resultante y el papel 
social que se le atribuye a través de los contenidos amplificados por la Sociedad de la Información

- Analizar el método de creación de audiencias previo al diseño del producto audiovisual que se soportará en un determinado uso del lenguaje cinematográfico, con la finalidad de generar necesidades, expectativas, emociones y /o empatías al público.

- Poner el valor la necesidad de adquirir la competencia audiovisual precisa tanto para emitir mensajes en el código audiovisual como la leerlo de modo correcto, apoyados en el espíritu crítico que permita, desde una perspectiva de género, el conocimiento de tal lenguaje, para distinguir el mensaje en sí de la intención con la que fue empleado.

- Identificar en el texto fílmico los arquetipos femeninos en que se basan los personajes de los cuentos maravillosos.

- Realizar una lectura guiada del texto fílmico con el público para ayudarles a distinguir la función generadora de empatías e identificaciones con los arquetipos femeninos previamente localizados.

- Reflexionar sobre el uso sexista del lenguaje cinematográfico tanto desde una percepción activa de este como desde la recepción pasiva.

\section{2. "Una nit". Un año en 16 minutos. Caperucita Roja o los comportamientos inadecuados}

Las dos proyecciones del cortometraje catalán "Una nit" (Bayarri 2014) cuyo desarrollo y conclusiones ocupan esta investigación, tuvieron lugar en el mes de 
noviembre de 2016, como una actividad educativa y formativa llevada a cabo en torno a los actos que diferentes instituciones y organismo desarrollan con motivo de la celebración del Día Internacional contra la Violencia de Género. En este caso, la actividad itinerante educativa que Fundación Inquietarte, en el seno de la Cátedra UNESCO Paz, Solidaridad y Diálogo Intercultural, lleva a cabo a través del festival internacional de cortometrajes, Visualízame.

La primera de las proyecciones tuvo lugar en horario de mañana (4 horas) en el IES Sierra Mágina de Huelma (Jaén), con alumnado de $4^{\circ}$ de la ESO y Bachiller de entre 14 y 18 años, un total de 50 participantes. La segunda, en horario de tarde (4 horas), en el local social de la asociación de mujeres Nuevas Formas de la localidad de Íllar en la provincia de Almería, con 12 mujeres de entre 50 y 70 años.

En la unidad didáctica "Una nit, un año en 16 minutos. Caperucita roja o los comportamientos inadecuados" se trabajan los siguientes contenidos:

- Breve introducción a la sociedad de la información.

Características. Consecuencias de la revolución tecnológica en los procesos comunicativos y educativos. La estructura del conocimiento en la sociedad de la información. Lenguaje cinematográfico. Generador de emociones: Elementos y usos. La necesidad de competencia audiovisual.

- Breve introducción a la literatura de la tradición oral. Los cuentos maravillosos. Arquetipos Femeninos. Identificación de arquetipos en la obra cinematográfica.

- La mujer en el audiovisual. La imagen de la mujer que los medios trasladan a la sociedad. 
- La violencia mediática consciente ejercida sobre la mujer y el peligro de su normalización

- El empleo aleccionador de los arquetipos.

- La lectura de la imagen: Caperucita roja o los comportamientos inadecuados.

Durante la sesión, tanto el alumnado del instituto como las mujeres de la asociación recibieron unos conocimientos teóricos mínimos e imprescindibles sobre la Sociedad de la Información, características y consecuencias en los procesos de comunicación (Pérez Tornero 2000: 35-37), sobre la gramática del lenguaje cinematográfico (Romaguera i Ramiò 1999: 19-38), nociones básicas de oralidad (Morote 2010: 139-150), de los personajes de los cuentos maravillosos (Propp 1985: 37120) y de sus significados arquetípicos (Bettelheim 2006: 160-284). Con posterioridad a la lectura del texto fílmico, se reflexionó con el público estudiantil sobre las distintas fases en las que se desarrolla un ciclo de maltrato (Madina 2005). Tras la sesión teórica tuvo lugar la fase práctica, consistente en la proyección del cortometraje "Una nit" (Bayarri 2014) sin una guía de lectura, invitando al alumnado a reconocer por sí mismo los conceptos de lenguaje cinematográfico recién adquiridos, sin ayudarle en su proceso de salida de la zona de confort en la que se encontraba como público pasivo para que, tras una segunda lectura, está ya guiada, distinguiera los distintos niveles de percepción a los que accedía en base a su competencia audiovisual, y las consecuencias de esta carencia.

Tras la identificación de los elementos del lenguaje cinematográfico, y la constatación del valor del audiovisual en la enseñanza como herramienta innovadora (Tynner 1993: 171-197), el alumnado analiza el valor arquetípico de los personajes y su función aleccionadora (Bettelheim 2006:160-169). 
2.1. Análisis del cortometraje "Una nit” (Bayarri 2014)

La realizadora catalana resultó finalista en la V edición del festival Visualízame, Audiovisual \& Mujer, y seleccionada para la actividad educativa itinerante que, entre ediciones, realiza el festival. "Una nit" formó parte de la selección del trabajo elegido para proyectar en las actividades temáticas organizadas por la lucha contra la Violencia de Género.

\subsubsection{Sinopsis}

Dos personajes anónimos, un hombre y una mujer, van a pasar la velada juntos en el apartamento del joven, después de haberse conocido en un bar. La relación que van entablando en los 16 minutos que dura el cortometraje reproducirá las diferentes etapas por las que atraviesa una relación de maltrato, de un modo tan sutil que hasta el giro final del guion, cuando el personaje del chico muestra su cara más violenta, el espectador no se da cuenta de que estamos ante un maltrato, y no lo ha percibido porque el guion se sustenta en conversaciones, gestos, situaciones que, la sociedad ha normalizado. Él, así se le identifica en los créditos, demuestra en el modo de tratarla que no le presta atención, que se burla de ella, que no la tiene en cuenta ni a ella ni a sus necesidades y que el hecho de que incumpla las normas que le ha impuesto sin que ella repare justifica un ataque de ira que conlleve una agresión violenta. Desde olvidar cuál es la bebida que ella prefiere, aunque la chica le recuerda que lo habían hablado en el bar, hasta que no le consienta marcharse cuando ella así lo desea, pasando por el intento de imponer una relación sexual en los términos que únicamente él estipula, en este caso, sin usar preservativo, será él hombre quien tenga en su mano el derrotero de la noche, 
mientras la joven, sin darse cuenta, cae en la trampa y busca la aprobación masculina constantemente. Tras el visionado del cortometraje, el alumnado y el colectivo de mujeres reciben la siguiente propuesta de reflexión:

\begin{tabular}{|l|c|}
\hline Violencia mostrada & Violencia representada \\
\hline Trama & Proceso de maltrato resumido en 16 minutos \\
\hline
\end{tabular}

Diferenciar entre estos dos tipos de violencia, cuando hasta apenas el giro final de guion la mayoría de ambos públicos no había distinguido ninguna es la primera dificultad con la que se enfrentaban.

Trabajando en el nivel de percepción básico en el que se encuentra los dos grupos, es decir, con lo percibido a través de los sentidos de la vista y el oído, solo les permitía asimilar información como público pasivo: la violencia con la que Él rompe el espejo del cuarto de baño tras enfadarse con Ella y la violencia con la que la inmoviliza y le tapa la boca con las manos para impedirle pedir ayuda. Existen otros momentos anteriores a este giro final en el que Él muestra su verdadero rostro, como los desprecios acompañados de despectivos gestos con los brazos que, normalizados, pasan desapercibidos al grupo de jóvenes, no así al de mujeres, si bien, tampoco ellas citan esos ejemplos al ser preguntadas por la violencia "mostrada". 
Tabla II. Violencia representada

\begin{tabular}{|r|c|}
\hline \multicolumn{1}{|c|}{ Concepto } & Hitos en el cortometraje \\
\hline Violencia representada & Proceso de maltrato resumido en 16 minutos \\
\hline & Rechazo = Búsqueda \\
\hline & + Rechazo = +Búsqueda \\
\hline & Dominio \\
\hline & De la manipulación al miedo \\
\hline
\end{tabular}

Las distintas etapas por las que pasa una relación de violencia machista, como indica Madina (2005) son tres: Escalada de la tensión: un paulatino incremento de la tensión por parte del hombre que podría derivar en una violencia física o no. Este crecimiento de la tensión lo mismo se produce a lo largo de varios meses que puede suceder en unos pocos minutos; Descarga de la violencia: una vez que el hombre se descarga contra la mujer, física o verbalmente, cesa la tensión y la violencia porque ya se ha desahogado; Y, por último, el hombre se muestra conciliador y amable y trata de recuperar la relación. En la mayoría de los casos, la mujer suele creerlo, incluso de modo voluntario y tiene a justificar la violencia achacándola a las circunstancias.

Este ciclo, con el tiempo, tiende a repetirse más frecuentemente y con los arranques de violencia cada vez más fuertes. De una mala respuesta, de un desplante gestual, se pasa a una agresión leve (bofetada, empujón) y después esta se ira recrudeciendo. El hombre puede llegar a vivir una habituación a la violencia que acabará por eliminar de la fórmula la fase del arrepentimiento. 
En "Una nit" las etapas se constatan, incluidos los ciclos de repetición:

Tabla III. Etapas de la violencia de género representadas en el corto

\begin{tabular}{|c|c|}
\hline Etapas & Hitos en el cortometraje \\
\hline \multirow[t]{2}{*}{ Tensión } & Desprecio (desconoce sus gustos) \\
\hline & Se burla de ella (cerveza) \\
\hline \multirow[t]{2}{*}{ Violencia } & Malos gestos \\
\hline & Elevaciones de la voz \\
\hline Ella busca aprobación & Acercamientos solicitando su perdón \\
\hline Tensión & La quiere obligar a tener sexo sin preservativo \\
\hline Violencia & Elevación de la voz y empujones \\
\hline Arrepentimiento de él & Él pide perdón \\
\hline \multirow[t]{2}{*}{ Tensión } & Rotura del espejo con el martillo \\
\hline & No hay arrepentimiento \\
\hline Aislamiento de la víctima & Ella enmudece y se esconde \\
\hline
\end{tabular}

Desde la primera muestra de desprecio hacia ella hasta que la inmoviliza impidiéndole gritar para pedir ayuda, las tres fases se van sucediendo a lo largo del cortometraje in crescendo. Victoria de él basada en el dominio. La víctima incurre en un comportamiento inadecuado y toma cuerpo el arquetipo de Caperucita Roja. Mucho 
antes de que Perrault publicara su colección de cuentos de hadas en 1697, los elementos literarios en los que se sustenta el famoso cuento de la niña devorada por el lobo ya cumplían una función amenazadora, en algunos casos también esperanzadora frente a la posibilidad de alejarse o incumplir la norma, así los hijos de Cronos, devorados por el padre hasta que la madre, Rea, consiguió salvar al menor de ellos, Zeus, sacándolo de las entrañas de Cronos y sustituyéndolo por una piedra (Méndez 2012: 43-45) o la historia latina de Egberto de Lieja, Fecunda ratis, quien en el siglo XI ya narraba las andanzas de una niña vestida de rojo que deambulaba en compañía de lobos (Paredes y Gracia 1989:111). No nos detendremos en los motivos por los que Perrault, al contrario que los hermanos Grimm, opta por dar la victoria al lobo, reforzando el valor atemorizador del relato, precisamente es la advertencia punitiva del mismo la que analiza desde el psicoanálisis Bettelheim (2006: 169-175) deteniéndose en lo que él denomina "la sexualidad incipiente (de Caperucita) para la que no está todavía emocionalmente madura" (2006: 182), inmadurez que la llevará a padecer la ambivalencia de querer actuar correctamente, en este caso, acatar la norma socialmente consensuada y aceptada, y el deseo de no hacerlo. En el cortometraje, "Una nit", ella desea transgredir dicha norma, atravesar el bosque prohibido de la mano de un desconocido. A partir de este acto de desobediencia, lo que le pueda acontecer es consecuencia de su comportamiento transgresor. El texto fílmico pues reproduce la finalidad aleccionadora del relato primitivo.

Las sesiones de proyección y análisis tuvieron lugar el 22 y el 28 de noviembre de 2015 respectivamente. La primera de ellas con jóvenes de entre 14 y 18 de edad, estudiantes de $4^{\text {o }}$ de ESO y Bachiller del IES Sierra Mágina de Huelma (Jaén). De ellos, 32 eran de sexo femenino y 18 de sexo masculino. La segunda, con 12 mujeres de entre 
50 y 70 años, pertenecientes a la asociación de mujeres Nuevas Formas de la localidad de Íllar (Almería)

Para evaluar el uso del audiovisual como herramienta de innovación didáctica, la deficiente competencia audiovisual de los espectadores, la normalización consecuencia de dicha incompetencia y el valor aleccionador de los arquetipos se llevó a cabo una encuesta cualitativa (Selltiz y Jahoda 1980: 132-164).

Las preguntas en la encuesta se dividían en cuatro bloques con preguntas relacionadas con la valoración del audiovisual como herramienta, con las diferencias en las lecturas del texto fílmico entre el primer pase, sin guía de lectura, y tras las reflexiones propuestas, el reconocimiento de violencias representadas y el reconocimiento del arquetipo respectivamente.

Tabla IV. Preguntas sobre la valoración del audiovisual como herramienta educativa. Alumnado de ESO y Bachiller (Huelma, Jaén)

\begin{tabular}{|c|r|}
\hline Preguntas & Respuestas \\
\hline ¿Te ha resultado interesante este tipo de clase & Sí : 50 \\
\hline con proyección de cortometraje? & No: 0 \\
\hline ¿Habías reconocido la violencia representada? & Sí: 7 \\
\hline Qué violencias mostradas has reconocido? & No: 34 \\
\hline
\end{tabular}




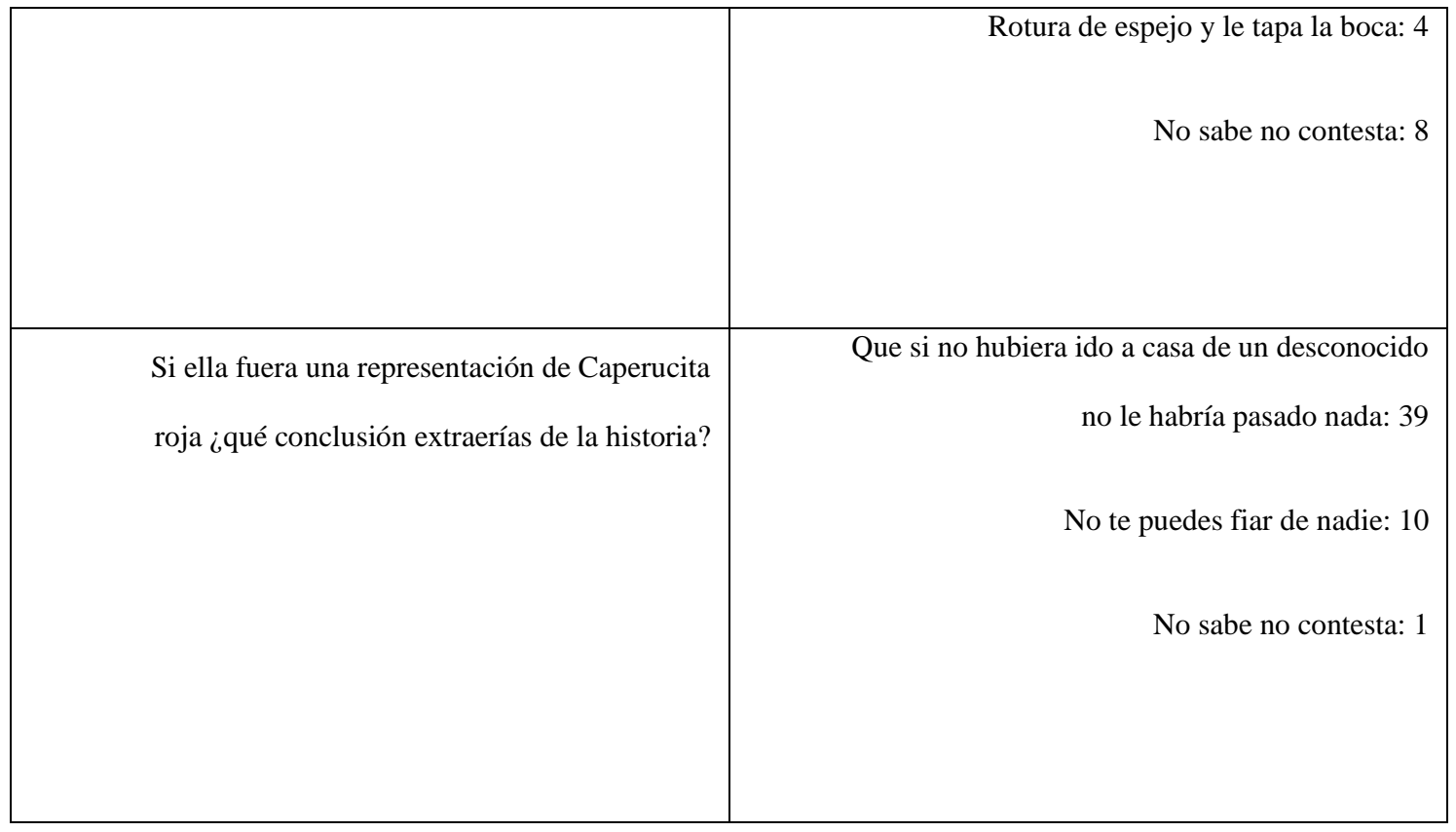

Tabla V. Preguntas sobre la valoración del audiovisual como herramienta educativa. Asociación de mujeres Nuevas Formas (Íllar, Almería)

\begin{tabular}{|c|r|}
\hline Preguntas & Respuestas \\
\hline con proyección de cortometraje? & Sí: 12 \\
\hline ¿Te ha resultado interesante este tipo de clase & No: 0 \\
\hline ¿Habías reconocido la violencia representada? 0 \\
\hline ¿Qué violencias mostradas has reconocido? & No: 12 \\
\hline Si ella fuera una representación de Caperucita & Que si no hubiera ido a casa de un desconocido \\
\hline
\end{tabular}




\begin{tabular}{|r|r|}
\hline roja ¿qué conclusión extraerías de la historia? & no le habría pasado nada: 9 \\
& No sabe no contesta: 3 \\
\hline
\end{tabular}

\section{Conclusiones}

La identificación de los dos grupos como públicos pasivos es general con el reconocimiento mayoritario de no haber trabajo el segundo nivel de la percepción que habría implicado el análisis de lo percibido. Además, el escaso reconocimiento de las violencias mostradas, aquellas de las que les llega información a través de un primer nivel perceptivo, refuerza la afirmación del poder normalizador del audiovisual en la comunicación.

De este modo, la ya reiterada omnipresencia de la sociedad de información con los nuevos lenguajes en los que se soporta, y la potencia de estos gracias a la revolución tecnológica, hacen necesaria e imprescindible la consecución de una competencia audiovisual que permita la subsistencia y el refuerzo de la mirada y el espíritu críticos. La normalización que propician los medios de comunicación en la sociedad de la información refuerza situaciones, roles y acontecimientos que lejos de finalizar con la violencia machista, la perpetúan. En cuanto a la función aleccionadora del arquetipo literario, en este caso el de Caperucita Roja, refuerza la normalización de que la violencia, es consecuencia de la imprudencia de la víctima más que de un ejercicio impune de la violencia por parte del hombre sobre la mujer, por el mero hecho de serlo, 
el valor atemorizador instaurado desde la masculinidad del orden social continúa en la huella cinematográfica arquetípica.

\section{Referencias bibliográficas}

Alba, Ramón. 1999. Literatura español. Una historia de cine. Madrid: Polifemo, Ministerio de Asuntos Exteriores, Dirección General de Relaciones Culturales y Científicas.

Bettelheim, Bruno. 2006. Psicoanálisis de los cuentos de hadas. Madrid: Crítica.

Camarero Gómez, Gloria. 2006. Adaptaciones de la literatura española en el cine español. Referencias y bibliografía. Alicante: Biblioteca Virtual Miguel de Cervantes. http://www.cervantesvirtual.com/nd/ark:/59851/bmcms437 [04/ 10/2014]

Cruz López, Yolanda. 2012. Literatura Oral y Cine. Intertextualidad y Didáctica. Trabajo de Investigación de Tercer Ciclo, inédito. Almería: Universidad de Almería.

2012. Oralidad y Cine. Narrador y relato: Tim Burton y los cuentos maravillosos. Memoria Diplomatura Inédita. Valladolid: Universidad de Valladolid.

Escudero, Miguel Ángel y Eva Isabel Cuesta. 2005. Cine y literatura española. Madrid: Comunidad de Madrid, Consejería de Cultura y Deporte. Dirección General de Promoción Cultural.

Heredero, Carlos F. y Carlos Santamarina. 2010. Biblioteca del cine español (Fuentes literarias 1900 - 2005). Madrid: Cátedra.

Gómez López, Nieves. 1998. Cuentos de transmisión oral del Poniente almeriense. Almería: Ayuntamiento de Roquetas de Mar.

Gómez López, Nieves y José Manuel Pedrosa. 2002. El canto de las sirenas. Almería: Ayuntamiento de Roquetas de Mar.

Gómez López, Nieves; Martínez, Manuela y José Manuel Pedrosa. 2007. La literatura de tradición oral del levante almeriense. Almería: Grupo de Desarrollo Rural del Levante Almeriense.

Gómez Mesa, Luis. 1978. La literatura española en el cine nacional, 1907 1977:(documentación y crítica). Madrid: Filmoteca Nacional. 
Madina, Javier. 2005. Perfil psicológico e intervención de los hombres maltratadores [en línea]. http://www.psicologo-barcelona.cat/pareja/fasesdel-maltrato/ [02/10/2016].

Méndez Mendalav, Xavier. 2012. Mitos que emergieron del sueño. Madrid: Bukok.

Morote, Pascuala. 2010. "Universalidad de la literatura Oral". Tradición y Modernidad de la Literatura Oral. Coords. Pedro G. Cerrillo Torremocha y César Sánchez Ortiz. Toledo: Ediciones de Castilla La Mancha. 139-150.

Paredes Núñez, Juan y Paloma García. 1989. Tipología de las formas narrativas breves románicas medievales. Granada: Universidad de Granada.

Parra, Carmen y Yolanda Cruz. 2015. "El cine como herramienta formativa en el aula: Educar la mirada". Docencia virtual y experiencias de innovación docente: entornos $b$ - learning y e- learning. Coords. M. Villca y A. Varreas. Huygens: Barcelona 267-278.

Pedrosa, José Manuel. 2002. La autopista fantasma. Madrid: Páginas de espuma.

Pérez Tornero, José Manuel. 2000. "Las escuelas y la enseñanza en la sociedad de la información”. Comunicación y Educación en la sociedad de la información. Comp. José Manuel Pérez. Barcelona: Paidós. 35-37.

Perrault, Charles; Jacob y Wilhem Grimm y Ludwig Tieik. 2012. Caperucita Roja. Madrid: Nórdica Libros.

Propp, Vladimir. 1985. La morfología del cuento. Madrid: Akal.

Quesada, Luis. 1986. La novela española y el cine. Madrid: Ediciones JC.

Romaguera i Ramiò, Joaquim. 1999. El lenguaje cinematográfico. Gramática, géneros, estilos y materiales. Madrid: De la Torre.

Selltiz, M., Jahoda, M., y, S.W. Deutsch. 1980. Métodos de investigación en las relaciones sociales. Madrid: Rialp.

Trejo Delabre, Raúl. "Vivir en la Sociedad de la Información. Orden global y dimensiones locales en el universo digital". CTS + I: Revista Iberoamericana de Ciencia, Tecnología, Sociedad e Innovación. Septiembre (2001). https://www.oei.es/historico/revistactsi/numero1/trejo.htm [20/10/2006]

Tynner, K. 1993. "Alfabetización audiovisual: el desafío del fin de siglo". La revolución de los medios audiovisuales. Coord. Roberto Aparici. Madrid: La torre. 171-197. 